\title{
A NOTE ON ALZER'S REFINEMENT OF AN ADDITIVE KY FAN INEQUALITY
}

\author{
PETER R. MERCER
}

Abstract. An elementary proof of Alzer's generalization of Ky Fan inequality is given.

Mathematics subject classification (1991): 26D15.

Key words and phrases: Ky Fan inequality, arithmetic mean, geometric mean.

\section{REFERENCES}

[1] H. AlZER, On an additive analogue of Ky Fan's Inequality, Indag. Mathem., N.S. 8 (1997), 1-6.

[2] E. F. BeCKenBaCh \& R. Bellman, Inequalities, Springer, Berlin, 1961.

[3] D. I. CARTWRIGHT \& M. J. Field, A refinement of the Arithmetic Mean - Geometric Mean Inequality, Proc. Amer. Math. Soc. 71 (1978), 36-38. 